\title{
Konsensustreffen Mammadiagnostik @ Akademie Online
}

In diesem Jahr bieten wir Ihnen im Rahmen der DRG-Fortbildungsreihe „Akademie Online“ eine ganz besondere 3-teilige Veranstaltung an: Das Konsensustreffen Mammadiagnostik ist zu Gast bei Akademie Online.

Die spannende, traditionsreiche und für Ihr Fachgebiet maßgebliche Veranstaltung, die bereits seit 2007 alle 2 Jahre unter der wissenschaftlichen Leitung von Prof. Dr. Markus Müller-Schimpfle in Frankfurt stattfindet, ist in diesem Jahr komplett in den digitalen Raum umgezogen und gestaltet 3 Schwerpunkttermine im Programmangebot von Akademie Online:

13. April 2021: 2D-/3D-Mammografie 04. Mai 2021: Mamma-MRT

08. Juni 2021: Mamma-Sonografie

In jedem der 3 Webinare werden aktuelle Fragestellungen der Mammadiagnostik diskutiert, und das im ganz wörtlichen Sinne: Nach einem Einführungsvortrag folgen jeweils ein Pro- und ein Kontra-Vortrag z. B. zu der Frage, ob die 3D-Tomosynthese mit 2D-Rekonstruktion die Standard-2D-Mammografie bereits heute ersetzen sollte oder eben nicht. Nach einer anschließenden Live-Paneldiskussion sind Sie als Teilnehmer*innen gefragt: Per TED-Abstimmung lassen Sie Ihre Meinung in das Stimmungsbild der Fachcommunity einfließen. In 2 weiteren Terminen werden die Fragen diskutiert, ob MRT und Ultraschall bei dichtem Brustgewebe standardmäßig eingesetzt werden sollten. 
Als Teil des Akademie-Online-Programms ist die Veranstaltungsreihe „Konsensustreffen Mammadiagnostik“ in diesem Jahr für alle Jahresabonnent*innen von Akademie Online inklusive - die Teilnahme, Login usw. funktionieren analog. Für alle, die kein Jahresabo haben, gibt es natürlich auch hier die Möglichkeit der Einzelbuchung der 3 Termine über den Programmkalender.
Wir freuen uns sehr auf dieses spannende Format und die kollegiale Diskussion der Themen, die uns im klinischen Alltag der Mammadiagnostik begleiten!

Alle Infos zu Programm und Anmeldung auf den Seiten von Akademie Online unter: www.drgakademie.de > Akademie Online

\section{Konsensustreffen Mammadiagnostik} @ Akademie Online

Wissenschaftliche Leitung:

Prof. Dr. Markus Müller-Schimpfle

Referent*innen:

Prof. Dr. Werner Bader

Prof. Dr. Pascal Baltzer

Dr. Maria Bernathova

Prof. Dr. Michael Fuchsjäger

Prof. Dr. Michael Golatta

Prof. Dr. Thomas Helbich

Prof. Dr. Sylvia Heywang-Köbrunner

Prof. Dr. Alexander Mundinger

Prof. Dr. Rüdiger Schulz-Wendtland

Prof. Dr. Katja Siegmann-Luz

Prof. Dr. Christine Solbach

Prof. Dr. Stefanie Weigel 